\title{
Linfangioma orbitário: relato de caso
}

\author{
Orbital lymphangioma: case report
}

\author{
Filipe José Pereira ${ }^{1}$ \\ Sabrina de Pellegrini Trindade ${ }^{2}$ \\ Antonio Augusto Velascoe Cruz ${ }^{3}$ \\ Thiago Prazeres Salum Müller ${ }^{4}$
}

Trabalho realizado no Departamento de Plástica Ocular do Hospital Governador Celso Ramos - Florianópolis (SC) - Brasil. Centro de Estudos de Oftalmologia Dr. Aramis Ritzmann Mendes.

${ }^{1}$ Chefe do Departamento de Plástica Ocular do Hospital Governador Celso Ramos - HGCR - Florianópolis (SC) - Brasil.

${ }^{2}$ Residente de Oftalmologia (2007) do HGCR - Florianópolis (SC) - Brasil.

${ }^{3}$ Professor Titular do Departamento de Oftalmologia, Otorrinolaringologia e Cirurgia de Cabeça e Pescoço da Faculdade de Medicina de Ribeirão Preto da Universidade de São Paulo - USP - Ribeirão Preto (SP) - Brasil.

${ }^{4}$ Residente de $2^{\circ}$ ano de Oftalmologia do HGCR - Florianópolis (SC) - Brasil.

Endereço para correspondência: Filipe José Pereira. CCPO - Clínica Catarinense de Plástica Ocular. Rua Dom Jaime Câmara, 179 - S1. 704/705 - Florianópolis (SC) CEP 88015-120

E-mail: filipeccpo@uol.com.br

Recebido para publicação em 13.10.2008

Última versão recebida em 26.07.2009

Aprovação em 22.09.2009

\section{INTRODUÇÃO}

Linfangioma é uma lesão veno-linfática incomum, também definido como hamartoma vascular de origem linfática, que cresce entre estruturas normais $^{(1-2)}$. O termo linfangioma é utilizado de forma apropriada quando há isolamento hemodinâmico, ou seja, a lesão não está relacionada com o sistema arterial ou venoso ${ }^{(2)}$, embora sejam propensos a hemorragia intrínseca de pequenos vasos, expandindo porções da cadeia vascular em grandes "cistos-chocolate"(3).

O linfangioma pode acometer apenas a conjuntiva e a pálpebra, localizar-se na órbita ou apresentar-se com os dois componentes, superficial e profundo $^{(2)}$.

Pode manifestar-se clinicamente por proptose, restrição da motilidade ocular extrínseca, ptose ${ }^{(4-7)}$, dor, diplopia ${ }^{(4,8)}$, exposição corneana ${ }^{(4)}$, perda visual por neuropatia óptica compressiva e ambliopia ${ }^{(7)}$. Exacerbações do quadro clínico tendem a ocorrer em casos de hemorragia intralesional e episódios de infecção das vias aéreas superiores, que resultam em hiperplasia linfóide por reação do sistema imunológico, o que explicaria a reativação do quadro ${ }^{(2,9)}$. O aumento da pressão intraocular pode causar náuseas e vômitos, e a estimulação vagal excessiva pode resultar em bradicardia, decorrente de reflexo óculo-cardíaco.

Varizes orbitárias, celulite orbitária e rabdomiossarcoma são diagnósticos diferenciais a serem considerados. A evidência de não continuidade da 
lesão com o sistema vascular diferencia o linfangioma de varizes. Em casos em que não se observa resolução ou em que houver progressão do quadro clínico, a biópsia deve ser considerada, a fim de descartar a possibilidade de rabdomiossarcoma.

Histologicamente constituem espaços revestidos por endotélio, não encapsulados, o que predispõe a recidivas após ressecção cirúrgica ${ }^{(1-2,10)}$.

Estudo histoquímico revelou que a composição celular do endotélio do linfangioma é diferente daquele que compõe os capilares $^{(4)}$, o que reforça a hipótese de o tumor ser uma entidade verdadeira da órbita e não uma má-formação vascular.

Este relato de caso está sendo apresentado com o objetivo de ressaltar a importância do linfangioma como diagnóstico diferencial de proptose na infância.

\section{RELATO DE CASO}

KA, nove anos, feminina, branca, procedente de Canelinha/SC, foi encaminhada por apresentar tumor orbitário à direita (Figura 1A), há dois anos. O quadro se iniciou com discreta proptose à direita, sem outras queixas. Foi realizada tomografia computadorizada (Figura 1B), evidenciando lesão expansiva de pequeno tamanho, extraconal, medial, na órbita direita. Evoluiu com baixa de acuidade visual, dor local e aumento significativo da proptose nos meses subsequentes. Foi, então, internada em outro hospital e submetida à biópsia da lesão orbitária, sob anestesia geral. A descrição macroscópica e microscópica da peça cirúrgica revelava: "fragmento de mucosa com dimensões 0,4 x 0,3 x 0,2 cm, revestido por epitélio estratificado escamoso, sem atipias, com proliferação linfática no córion, em meio ao estroma conjuntivo frouxo; presença de vasos sanguíneos e moderado infiltrado inflamatório composto por monomorfonucleares; compatível com linfangioma" (Figura 1C). Devido à piora importante da proptose, a paciente foi encaminhada ao serviço de oncologia pediátrica, que iniciou corticoterapia oral (metilprednisolona $20 \mathrm{mg} / \mathrm{kg} / \mathrm{dia}$ ). Foi solicitada ressonância magnética, a qual demonstrou lesão expansiva de aspecto cístico, não infiltrativa, extraconal, com formação de nível líquido-líquido, com a possibilidade de hemorragia intralesional (Figura 1D). Após uma semana de internação sem melhora clínica, foi encaminhada para o Hospital Governador Celso Ramos - Florianópolis. Nesta ocasião a paciente apresentava proptose grave (exoftalmometria de $29 \mathrm{~mm}$ ), quemose intensa da conjuntiva inferior (Figura 1A), ausência de percepção luminosa no olho direito e fundoscopia mostrando borramento do disco óptico e aumento da tortuosidade dos vasos. Foi realizada, sob anestesia geral, punção de cisto orbitário via transconjuntival, com agulha 25 x 7 acoplada a seringa de $10 \mathrm{ml}$, com aspiração de $35 \mathrm{ml}$ de líquido "cor de chocolate" (Figura 1E). Devido à intensa quemose da conjuntiva inferior, com consequente alteração do posicionamento palpebral e ectrópio e prejuízo da lubrificação da superfície
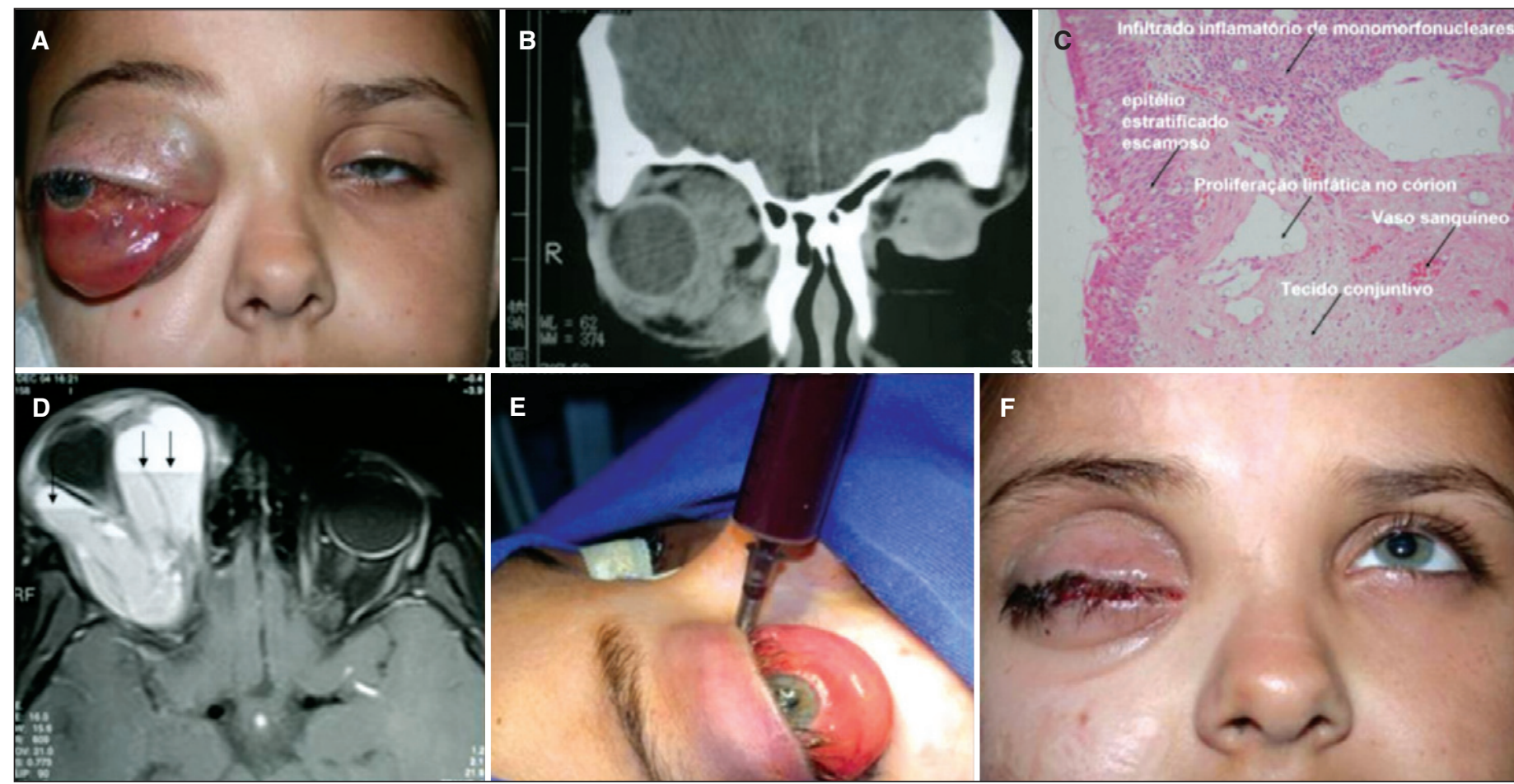

Figura 1 - A) Aspecto da face da criança no pré-operatório; B) TC inicial demonstrando massa tumoral irregular, heterogênea e infiltrativa em órbita direita; C) Aspecto anátomo-patológico de mucosa, revestido por epitélio estratificado escamoso, com proliferação linfática no córion, em meio a estroma conjuntivo frouxo, presença de vasos sanguíneos e moderado infiltrado inflamatório de monomorfonucleares compatível com linfangioma; D) Ressonância Magnética em T1 após biópsia, demonstrando hemorragia intralesional; E) Punção de cisto orbitário via transconjuntival usando seringa de $10 \mathrm{ml}$, com extração de grande quantidade de líquido sanguinolento; F) Tarsorrafia provisória imediatamente após a punção 
ocular, foi optado por tarsorrafia provisória (Figura 1F). O líquido aspirado foi classificado citologicamente como hemorrágico, sem contaminação bacteriana. No pós-operatório, a paciente evoluiu com resolução completa da proptose, da quemose e da dor. A corticoterapia foi suspensa, com redução progressiva da dose até o $15^{\circ}$ dia pós-operatório.

Após período de dois meses de melhora total da proptose, houve nova proptose por ressangramento espontâneo dos cistos, inclusive na porção anterior, causando novamente ectrópio mecânico. $\mathrm{O}$ tratamento realizado foi a exérese dos cistos orbitários, por meio do acesso lateral (cantólise e retirada de parede orbitária lateral a qual não foi recolocada para efeito de descompressão) e inferior (transconjuntival inferior). Houve, depois disso, regressão completa do quadro clínico (Figura 2).

Após oito meses, reiniciou quadro de proptose progressiva, novamente por ressangramento de lesão prévia, confirmada à tomografia computadorizada. Foi realizada nova cirurgia de ressecção de linfangiomas, usando o acesso transconjuntival inferior. A última avaliação foi realizada após dois meses, não havendo nenhuma queixa e estando a paciente sem proptose. Entretanto, tendo em vista que novos episódios podem ocorrer, foi orientada a respeito disso.

\section{DISCUSS ÃO}

A partir do exposto, ficou comprovado que a paciente em questão apresenta linfangioma orbitário. Trata-se de paciente do sexo feminino, que é discretamente mais acometido por linfangioma que o masculino, na proporção de $1,4: 1^{(2,5)}$.

A idade de aparecimento dos sintomas também coincidiu com a relatada na literatura, que é na primeira década de vida, embora o tumor possa manifestar-se desde o nascimento até a idade $\operatorname{adulta}^{(2)}$.

A imagem tomográfica típica do linfangioma é de padrão cístico e mal definido ${ }^{(2)}$, como observado na primeira tomografia realizada; e a progressão dos sinais e sintomas observados caracterizaram um processo de exacerbação provocado por hemorragia intralesional, confirmada com a ressonância magnética, quando foi possível observar o nível líquido-líquido, com volume proporcional à quantidade do material aspirado.
A corticoterapia sistêmica está indicada na ocorrência de proptose dolorosa, perda de visão ou alteração de motilidade. O corticóide atua reduzindo o efeito de massa da hipertrofia linfóide estabiliza a vasculatura e promove a involução da vascularização anormal; é uma opção de tratamento único ou como adjuvante em cirurgias. Comprovadamente o corticóide sistêmico diminui o tempo de duração dos sintomas e é mais responsivo em crianças $^{(8)}$, fato que não ocorreu em nossa paciente.

Tratamentos alternativos têm sido descritos, como a injeção intralesional de OK-432 (Streptococcus pyogenes do grupo A de origem humana), a qual foi realizada em um caso descrito na literatura com sucesso, porém apresentando algumas intercorrências, como aumento da pressão intraocular, febre, reação inflamatória local, sendo necessários mais estudos para a implantação desta prática na terapêutica corrente do linfangioma ${ }^{(6)}$.

A perda visual pode ser atribuída à compressão por tempo prolongado do bulbo ocular e do nervo óptico pelo tumor. A opção cirúrgica respeitou as indicações existentes, as quais são: proptose grave, exposição corneana, dor e congestão intensa da órbita ${ }^{(9)}$. Dentre as possibilidades cirúrgicas, destacamos a ressecção tumoral e, quando insuficiente, associar descompressão óssea da órbita para aumento do continente. Porém, na maioria dos casos, o tratamento conservador é o único necessário ${ }^{(7,9)}$. Apesar de não haver prognóstico de melhora visual, o resultado final foi satisfatório em relação ao aspecto estético.

\section{CONCLUSÃO}

O linfangioma é uma doença desafiadora, de difícil tratamento, com potenciais complicações visuais e estéticas, além da possibilidade de recidivas frequentes, como se pode constatar pelo caso aqui exposto.

\section{ABSTRACT}

A case of orbital lymphangioma in a 9 year-old female, with proptosis $($ Hertel $=29 \mathrm{~mm}$ ), ocular motility restriction, pain
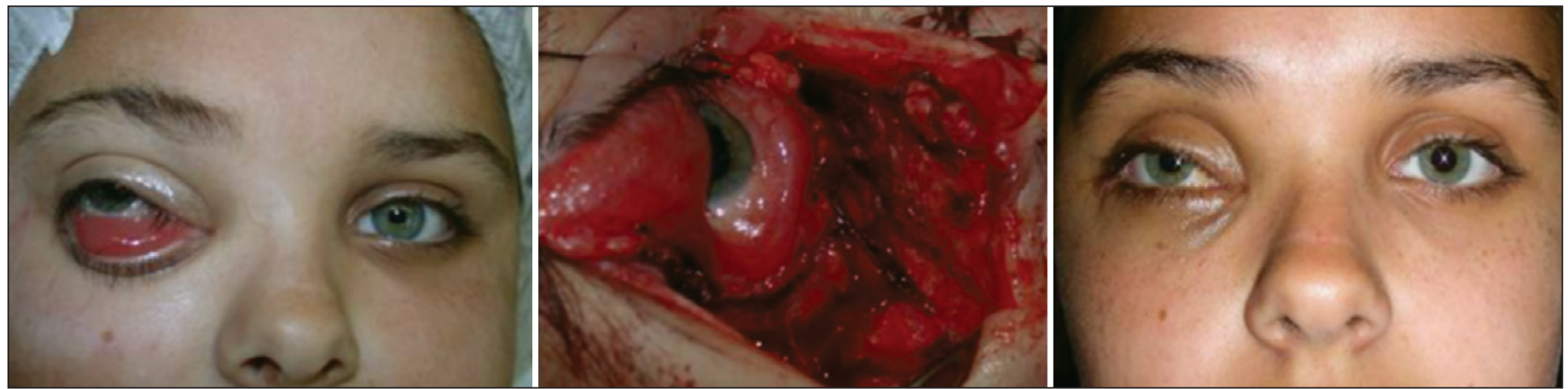

Figura 2 - Aspecto pré, trans e pós-operatório de criança portadora de linfangioma de órbita 
and visual loss due to compressive optic neuropathy is described. A magnetic resonance imaging (MRI) exam showed an expansive cystic lesion in the extraconal space of the right orbit, non-infiltrating with intralesional hemorrhage. The patient had no improvement with steroid treatment. A transconjunctival puncture with $35 \mathrm{ml}$ aspiration of a "chocolate color" fluid (which was confirmed by cytologic study as a hemorrhagic fluid) was performed. Exacerbations occurred after an intralesional hemorrhage (pain and increase of proptosis). The lesion was excised via lateral cutaneous approach (a lateral canthotomy and removal of the right orbital wall was performed done for decompression) and lower transconjunctival approach. Conclusion: Lynphangioma is a challenging disease and difficult to treat, with potential visual and cosmetic complications and the possibility of frequent recurrences.

Keywords: Lymphangioma/surgery; Orbital neoplasms/surgery; Lymphatic vessel tumors; Case reports

\section{REFERÊNCIAS}

1. Schoen FJ. Vasos sanguíneos. In: Cotran RS, Kumar V, Robbins SL, Schoen FJ, Patologia estrutural e funcional. $5^{\mathrm{a}}$ ed. Rio de Janeiro: Guanabara Koogan; 1996. p.453-4

2. Tucker SM. Vascular lesions of the orbit. In: Duane's clinical ophthalmology [CD-ROM]. Philadelphia: Lippincott-Raven; 2000.

3. Dutton JJ, Byrne SF, Proia AD, editors. Diagnostic atlas of orbital diseases Philadelphia: WB Saunders; 2000.

4. Hsuan J, Malhotra R, Davis G, Selva D. Orbital descompression for gross proptosis associated with orbital lymphangioma. Ophthal Plast Reconstr Surg. 2004; 20(6):463-5.

5. Tunç M, Sadri E, Char DH. Orbital Lymphangioma: an analysis of 26 patients Br J Ophthalmol. 2000;84(1):124-5.

6. Suzuki Y, Obana A, Gohto Y, Miki T, Otuka H, Inoue Y. Management of orbital lymphangioma using intralesional injection of OK-432. Br J Ophthalmol. 2000;84(6):614-7.

7. Kalisa P, Van Zieleghem B, Roux P, Meire F. Orbital lymphangioma: clinical features and management. Bull Soc Belg Ophthalmol. 2001;(282):59-68.

8. Sires BS, Goins CR, Anderson RL, Holds JB. Systemic corticosteroid use in orbital lymphangioma. Ophthal Plast Reconstr Surg. 2001;17(2):85-90.

9. Garza G, Fay A, Rubin P. Treatment of pediatric vascular lesions of the eyelid and orbit. Int Ophthalmol Clin. 2001;41(4):43-55.

10. Goto H, Usui M, Okada S. Histopathological study of orbital lymphangioma in an infant. Jpn J Ophthalmol. 2004;48(6):594-7.

\section{III \\ X Congresso da Sociedade Caipira de Oftalmologia}
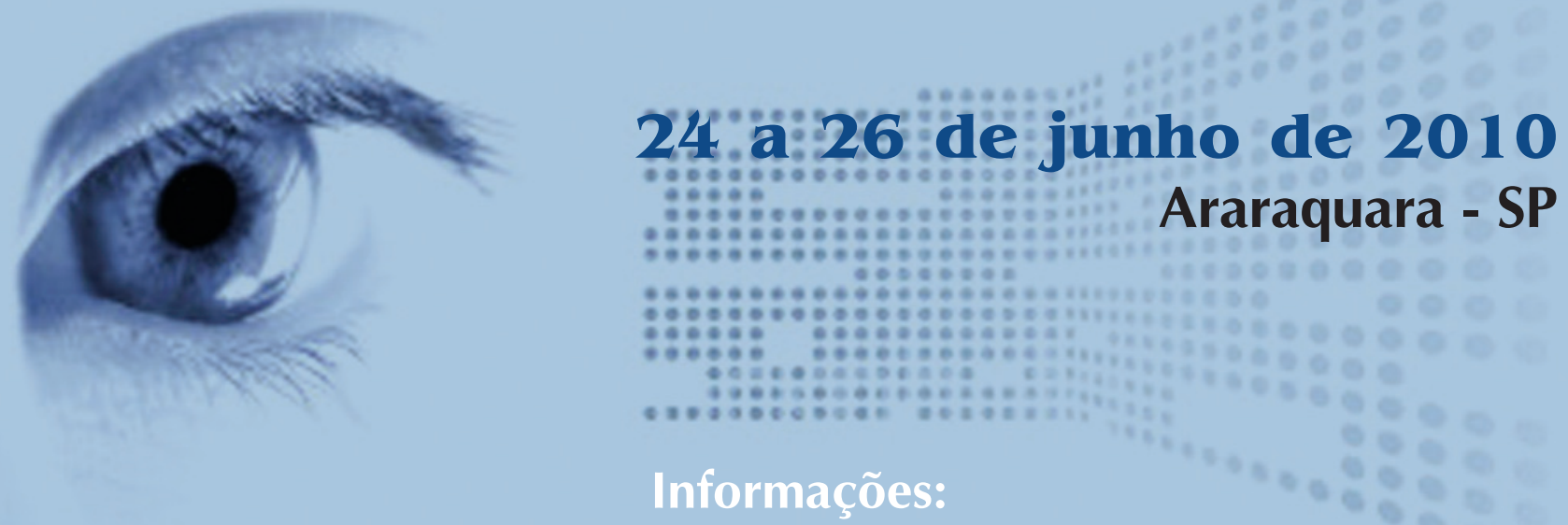

Tel.: (17) 3235-7017

e-mail: contato@sociedadecaipira.com.br

home page: http://www.sociedadecaipira.com.br/home/ 